\title{
'Need of the Hour': Early Diagnosis and Management of Multidrug Resistant Tuberculosis of the Spine: An Analysis of 30 Patients from a "High Multidrug Resistant Tuberculosis Burden” Country
}

\author{
Justin Arockiaraj ${ }^{1}$, Rajiv Karthik ${ }^{2}$, Joy Sarojini Michael ${ }^{3}$, Rohit Amritanand ${ }^{1}$, \\ Kenny Samuel David ${ }^{1}$, Venkatesh Krishnan ${ }^{1}$, Gabriel David Sundararaj ${ }^{1}$ \\ ${ }^{1}$ Spinal Disorder Surgery Unit, Department of Orthopaedics, Christian Medical College and Hospital, Vellore, India \\ ${ }^{2}$ Infectious Diseases Unit, Department of Medicine, Christian Medical College and Hospital, Vellore, India \\ ${ }^{3}$ Department of Microbiology, Christian Medical College and Hospital, Vellore, India
}

Study Design: Retrospective study.

Purpose: To report the prevalence of patients with multidrug-resistant (MDR) tubercular spondylodiscitis and their outcomes. Additionally, to assess the role of Xpert MTB/RIF assay in early detection of MDR tuberculosis.

Overview of Literature: MDR tuberculosis is increasing globally. The World Health Organization (WHO) has strongly recommended Xpert MTB/RIF assay for early detection of tuberculosis.

Methods: From 2006 to 2015, a retrospective study was conducted on patients treated for MDR tuberculosis of the spine. Only patients whose diagnosis was confirmed using either culture and/or the Xpert MTB/RIF assay were included. Diagnostic method, treatment regimen, time taken to initiate second-line antituberculosis treatment (ATT), drug-related complications, and cost of medications were analyzed. All patients with MDR were treated according to the WHO recommendations for 2 years. The outcome parameters analyzed included clinical, biochemical, and radiological criteria to assess healing status.

Results: From 2006 to 2015, a total of 730 patients were treated for tubercular spondylodiscitis. Of those, 36 had MDR tubercular spondylitis (prevalence, $4.9 \%$ ), and three had extremely drug resistant tubercular spondylitis (prevalence, $0.4 \%$ ). In this study, 30 patients, with a mean age of 29 years and a mean post-treatment follow-up of 24 months, were enrolled. The majority (77\%) had secondary MDR, 17 (56\%) underwent surgery, and 26 (87\%) completed treatment for 2 years and were healed. Drug-related complications (33\%) included ototoxicity, hypothyroidism, and hyperpigmentation of the skin. The average time taken for initiation of second line ATT for MDR patients with Xpert MTB/RIF assay as the diagnostic tool was 18 days, when compared to patients for whom the assay was not available which was 243 days.

Conclusions: The prevalence of MDR tubercular spondylodiscitis was 4.9\%. In total, $87 \%$ of patients were healed with adequate treatment. The sensitivity and specificity of the Xpert MTB/RIF assay to detect MDR was $100 \%$ and $92.3 \%$, respectively.

Keywords: Multi drug resistance; Tuberculosis; Diagnosis; Treatment

Received Mar 23, 2018; Revised Jun 20, 2018; Accepted Jul 16, 2018

Corresponding author: Justin Arockiaraj

Spinal Disorders Surgery Unit, Department of Orthopaedics, Christian Medical College and Hospital, Vellore, Tamil Nadu 632004, India Tel: +91-9443114380, +91-416-2282020, Fax: ++91-416-2232054, E-mail: svjustin.arockiaraj@gmail.com 


\section{Introduction}

Multidrug-resistant tuberculosis (MDR-TB) is alarmingly on the rise, specially in developing countries. In 2016, the World Health Organization (WHO) had declared 30 countries as 'high MDR-TB burden' countries [1]. According to the 2017 WHO report [2], 600,000 new cases of tuberculosis with resistance to rifampicin (RR-TB) were notified in 2016, of which 490,000 had MDR-TB. Almost half (47\%) of these cases were from India, China, and the Russian Federation. Globally, an estimated 4.1\% of new cases and 19\% of previously treated cases in 2016 had MDR/RR-TB. The percentage of patients in India with either MDR-TB or RR-TB is estimated to be $2.8 \%$ among new TB cases and $12 \%$ among previously treated cases [2].

Musculoskeletal tuberculosis often poses diagnostic challenges because of its chronic and indolent nature. The rise in the number of cases of MDR tubercular spondylodiscitis has resulted in the necessity for early diagnosis of this disease. In 2015, WHO recommended Xpert MTB/ RIF assay (Xpert; Cepheid, Sunnydale, CA, USA) [3] to detect extra pulmonary tuberculosis. However, to date, few studies reporting MDR tubercular spondylodiscitis have been published in English literature [4,5]. This study was undertaken with the objectives to report the prevalence of MDR tubercular spondylodiscitis at our tertiary care center and the outcomes of patients treated with second-line antituberculosis treatment (ATT). Secondly, to assess the role of Xpert MTB/RIF assay in detecting RRTB.

\section{Materials and Methods}

This was a retrospective study conducted from 2006 to 2015 (9 years) in our tertiary care center on all patients who were treated for multidrug-resistant tubercular spondylodiscitis. Only patients with proven MDR-TB (defined in Table 1) with either a positive culture and drug susceptibility test and/or a positive Xpert RIF/MTB assay were enrolled. Patients with other infective spondylodiscitis (pyogenic, fungal, etc.), those with inadequate follow-up, and those who did not provide consent for the study were excluded.

\section{Parameters studied}

Demographic profiles of the patients, including age, sex, and residence details, were collected. History of contact with tuberculosis, previous infection with tuberculosis, and prior treatment with anti-tuberculous chemotherapy (dosage and duration) was recorded. Severity of pain (assessed by Visual Analog Scale [6]), deformity (measured using Konstam's method by Konstam and Blesovsky [7]), neurological status of the patient (assessed using the American Spinal Injury Association [ASIA] Impairment Scale [8]), and the ability to bear weight or sit comfortably were assessed. Blood parameters included erythrocyte sedimentation rate (ESR), C-reactive protein (CRP), and liver function tests. Plain radiographs and magnetic resonance imaging (MRI) of the affected region were performed prior to and after completion of the treatment.

The indications for surgery included, substantial mechanical instability due to vertebral destruction [9], neurological deficits (ASIA A, B, and C), and deformity $>40^{\circ}$.

All patients were treated with five groups of second-line ATT for a period of 24 months according to drug susceptibility tests and WHO recommendations for pulmonary MDR tuberculosis [10,11]. Drug-related complications and cost of medications were also assessed.

Patients were followed up every 2 months for first 6 months, followed by quarterly visits for the rest of the first year, and finally twice yearly until the completion of the therapy. Annual follow-ups occurred thereafter.

Outcome analyses included clinical, biochemical, and radiological criteria to assess the healing status. Clinical parameters included pain, healing of sinuses, and return to work, as per MacNab's criteria [12]. Serological markers included decrease in ESR and CRP levels. Radiological criteria for healing included the presence of remineralization and reappearance of bony trabeculae, sharpening of articular margin, sharpening of cortical margin, sclerosis of vertebral bodies and end plates, and the fusion of vertebral bodies as revealed by plain X-rays. MRI criteria for healing included: the complete absence of paravertebral collection, complete resolution of enhanced vertebral body, and fatty replacement of marrow [4]. Thyroid function tests, audiometry, liver and renal function tests were performed to consider possible drug-related adverse effects.

\section{Results}

During the 9-year study period, a total of 730 patients were treated for tubercular spondylodiscitis at our tertiary 
care center, of which 36 had MDR tubercular spondylodiscitis. One patient died because of septicemia and five were lost during follow-up. As a result, 30 patients were enrolled in the study (Fig. 1). The mean age of patients

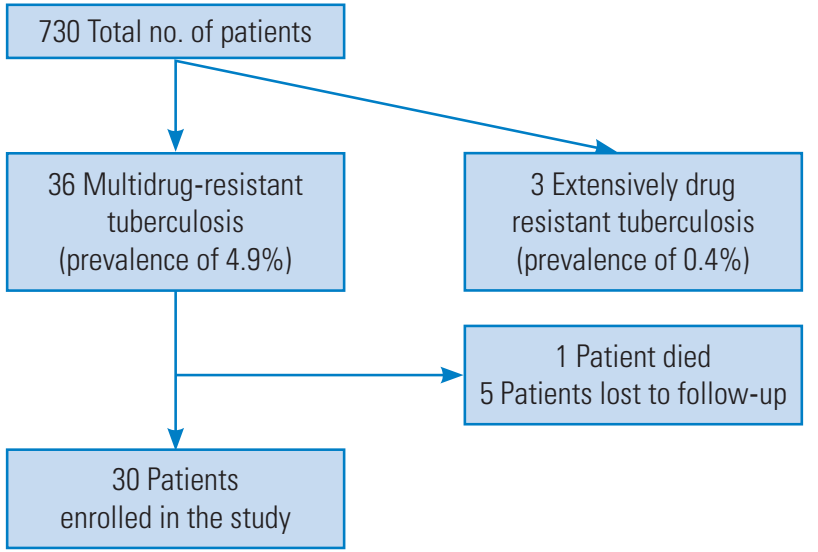

Fig. 1. Details of the study population. was 29 years (range, 5-56 years) and mean post-treatment follow-up was 24 months. The cohort had 14 men and 16 women, and six of them were children. Geographically, $63 \%$ were from the eastern states of India and 26\% were from the northeastern states of India. The majority (77\%) of the patients had history of being treated with first-line ATTs for an average period of 6-7 months. Radiologically, 23 patients $(76.7 \%)$ had para-discal involvement and seven patients (23.3\%) had vertebral involvement. Multicentric vertebral involvement was seen in seven patients (23\%). There was no preponderance among affected spinal levels. The dorsal, lumbar, or dorso-lumbar vertebrae were affected without a significant pattern.

Only 17 patients (56\%) underwent surgery, whereas the remainder had biopsies to confirm the diagnosis. Of the 17 patients, 15 underwent surgical decompression of the cord using the 'extended posterior circumferential decompression (EPCD)' approach [13]. Among the patients

Table 1. Definitions relating to TB and resistance patterns

\begin{tabular}{|c|c|}
\hline & Definitions \\
\hline Mono-resistance & Resistance to one first-line anti-TB drug only \\
\hline Poly resistance & Resistance to more than one first-line anti-TB drug (other than both isoniazid and rifampicin) \\
\hline Multidrug resistance & Resistance to at least both Isoniazid and rifampicin \\
\hline Extensively drug resistance & $\begin{array}{l}\text { Resistance to any fluoroquinolone and to at least one of the three second-line injectable drugs (capreomycin, } \\
\text { kanamycin, and amikacin), in addition to multidrug resistance }\end{array}$ \\
\hline Rifampicin resistance & $\begin{array}{l}\text { Resistance to rifampicin detected using phenotypic or genotypic methods, with or without resistance to other anti- } \\
\text { TB drugs. It includes any resistance to rifampicin, whether mono-resistance, multidrug resistance, poly-drug } \\
\text { resistance or extensive drug resistance }\end{array}$ \\
\hline Primary resistance & $\begin{array}{l}\text { Patients may be erroneously labelled as having primary resistance if they do not disclose previous treatment for } \\
\text { TB. }\end{array}$ \\
\hline Acquired resistance & $\begin{array}{l}\text { Patients who fail treatment (and are therefore labelled to have acquired resistance) may have been infected with a } \\
\text { resistant strain from the beginning or acquired resistance during treatment. }\end{array}$ \\
\hline
\end{tabular}

TB, tuberculosis.

Table 2. Final results following treatment for multidrug-resistant tuberculosis

\begin{tabular}{lll} 
Variable & \multicolumn{1}{c}{ Preoperative status } & \multicolumn{1}{c}{ Postoperativee status } \\
Neurological status & $\begin{array}{l}\text { Cauda equina syndrome (1 patient) } \\
\text { Paraplegics } \\
\text { (3 patients with ASIA A \& } 6 \text { patients with ASIA B) }\end{array}$ & $\begin{array}{l}\text { Remained the same and he is on bladder and bowel rehabilitation. } \\
\text { 3 Patients with ASIA A improved to ASIA C }\end{array}$ \\
& 6 Patients with ASIA B improved to ASIA D
\end{tabular}

ASIA, American Spinal Injury Association; VAS, Visual Analog Scale; ESR, erythrocyte sedimentation rate; CRP, C-reactive protein. 
who had surgery, $60 \%$ had neurological deficits, $60 \%$ had significant spinal deformity, and all of them presented with mechanical instability. In total, 26 patients (87\%) completed 2 years of therapy and were found to have both clinical and radiological evidence of healing. The remaining four patients are presently on MDR treatment. The results of treated patients have been formulated in Table 2 .

\section{Role of Xpert MTB/RIF assay in multidrug-resistant}

The Xpert MTB/RIF assay is a polymerized chain reaction test to diagnose tubercular spondylodiscitis and RR. In 2012, it was made available at our center. Therefore, of the 30 patients in the study group, only 13 had the diagnosis confirmed using the Xpert MTB/RIF assay. Only patients treated from January 2012 were included in the analysis to detect the role of Xpert MTB/RIF assay to diagnosis Rifampicin resistance. In total, 112 patients were culture positive for tuberculosis using Lowenstein-Jensen solid media for tubercular bacilli. Out of the 112 patients, 99 had culture sensitive tuberculosis, and 13 had MDR-TB.

Among the 13 patients with MDR-TB, 12 were revealed to have RR by the Xpert MTB/RIF assay. Sensitivity and specificity of the Xpert MTB/RIF assay to detect RR was determined to be $100 \%$ and $92.3 \%$, respectively (Table 3 ).

\section{Initiation of second-line antituberculosis treatment}

The time duration between their presentation to the hospital and the initiation of second-line ATT for patients with MDR-TB was 18 days on average when the Xpert MTB/RIF assay was used as the initial diagnostic tool. In patients without an Xpert MTB/RIF assay diagnosis, the time taken to initiate second-line ATT was 243 days since presentation.

\section{Drug-related complications}

Drug-related complications occurred in $33.3 \%$ (10 of
30) patients, with a total of 14 recorded events. The most common complications were hypothyroidism due to ethionamide (36\%) and aminoglycoside induced ototoxicity (28\%). Other toxicities included linezolid-induced peripheral neuropathy and optic neuritis, clofazimineinduced hyperpigmentation, and para-amino salicylic acid-induced severe gastritis. Of 30 patients, $10 \%$ had to discontinue medications because of adverse effects.

\section{Cost of second-line antituberculosis treatment}

The cost of medications for patients treated with firstline chemotherapy was on an average Rs. 3,648 (\$53) for a period of 9 months. The cost of patients treated with 2 nd line chemotherapy for MDR for a period of 2 years was on an average Rs. 2,57,000 (\$3775).

\section{Illustration 1}

A 50-year-old man presented with low backache for a period of 6 months. There was no history of contact with tuberculosis. Clinically, there was no neurological deficit. Plain radiography revealed sacralization of the L5 vertebra and erosion at the upper end plate of the L3 vertebra (Fig. 2A). The L2/3 disk space was reduced. MRI revealed an intraosseous abscess at the $\mathrm{L} 2 / 3$ vertebrae with associated involvement of the L1/2 and L2/3 disc spaces (Fig. $2 \mathrm{~B})$. Ultrasound guided aspiration of the psoas abscess revealed MDR tuberculosis. The patient was initiated with second-line ATT, and following a 2-year follow-up, plain radiography revealed sclerosis of the involved vertebrae with a positive 'sentinel sign'-bony fusion was observed at the $\mathrm{L} 2 / 3$ level (Fig. 2C).

\section{Illustration 2}

A 35-year-old woman presented with complaints of pain in the mid back and weakness in the lower limbs for a period of 1 month. One year prior, she had been treated with

Table 3. Rifampicin resistance and culture positivity

\begin{tabular}{lrrr} 
& Culture: rifampicin sensitive & Culture: rifampicin resistant & Total \\
Xpert MTB/RIF assay sensitive & 99 & 1 & 100 \\
Xpert MTB/RIF assay resistant & 0 & 12 & 12 \\
\hline Total & 99 & 13 & 112 \\
\hline
\end{tabular}

Sensitivity=100\%; specificity=92.3\%. 


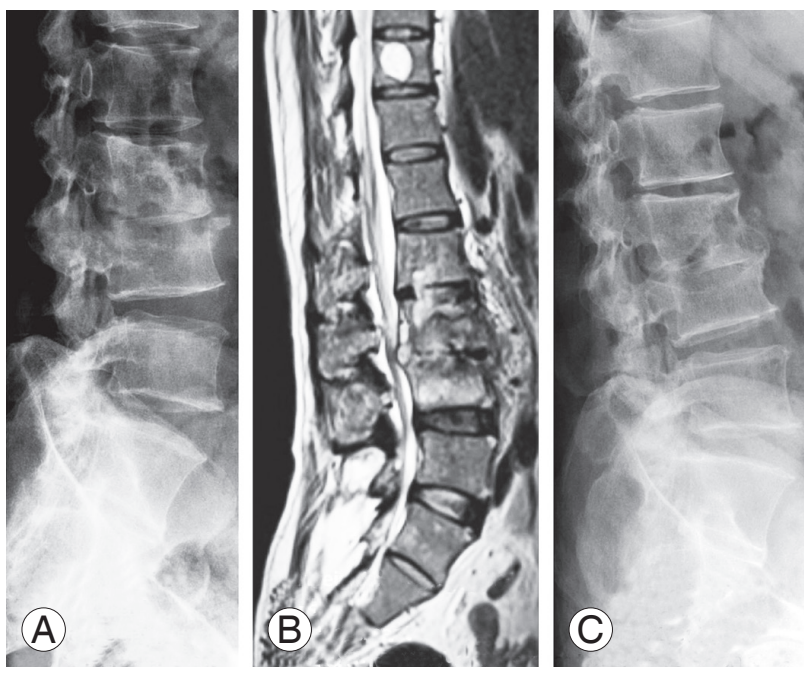

Fig. 2. (A) Plain radiography shows erosion in the upper end plate of the $\mathrm{L} 3$ vertebra with reduction in the $\mathrm{L} 2 / 3$ disk space. The $\mathrm{L} 5$ vertebra was sacralized. (B) MRI revealed an intraosseous abscess at the $L 2 / 3$ vertebrae involving the $L 1 / 2$ and $L 2 / 3$ disc spaces with a psoas abscess. (C) Plain radiography shows sclerosis of the involved vertebrae. Bony fusion occurred at L2/3. MRI, magnetic resonance imaging; TB, tuberculosis; ASIA, American Spinal Injury Association.
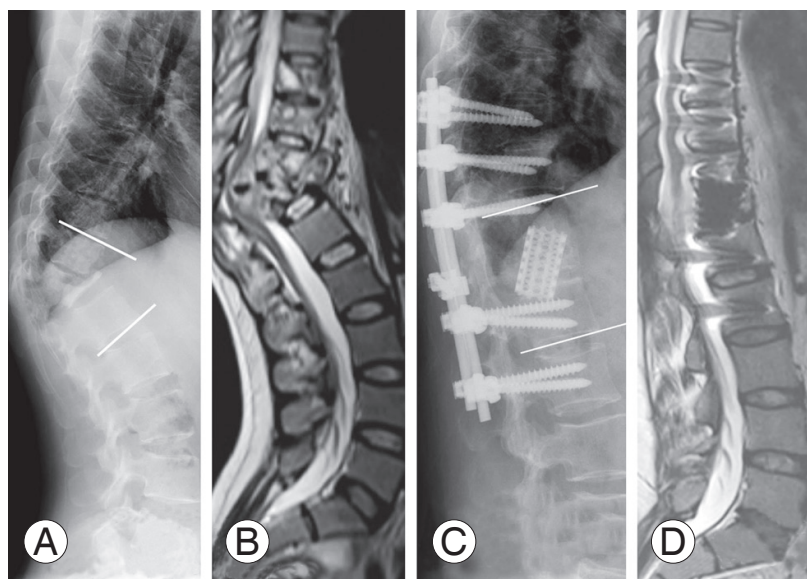

Fig. 3. (A) Plain radiography reveals near complete destruction of the D11 and D12 vertebrae with a $69.9^{\circ}$ kyphosis. (B) T2-weighted sagittal MRI shows complete destruction of the D11 and D12 vertebrae, as well as erosion of the D10 vertebra. An intraosseous abscess extending into the canal causes significant impingement of the spinal cord. (C) Plain radiography shows the anterior column reconstructed with cage and bone graft, sclerosis of the vertebra, healing of the disease, and deformity corrected to $4.9^{\circ}$ kyphosis. (D) T2-weighted sagittal MRI reveals an almost complete resolution of the abscess. MRI, magnetic resonance imaging; ASIA, American Spinal Injury Association.

ATT for pulmonary tuberculosis. Clinically, she had a gibbus deformity of the mid back extending over the spinous processes from D10 to L1. Furthermore, she had an ASIA B paraplegia with grade 0 motor power and decreased peri-anal sensation. Plain radiography revealed near com- plete destruction of the D11 and 12 vertebrae with a $69.9^{\circ}$ kyphosis (Fig. 3A). A T2-weighted sagittal MRI revealed complete destruction of the D11 and 12 vertebrae with additional erosion observed in the D10 vertebra (Fig. 3B). An intraosseous abscess extended into the epidural space leading to significant impingement of the spinal cord. The patient underwent circumferential decompression of the cord via the 'EPCD' approach. After thorough debridement and decompression of the cord, the anterior defect was reconstructed with a titanium mesh cage filled with morselized autogenous cancellous bone graft. Intraoperative samples revealed MDR tuberculosis. The patient was initiated on second-line ATT according to drug sensitivity testing. She gradually improved to ASIA E over a period of 4-6 months. Fig. 3C and D show the postoperative Xray and MRI, respectively, with $4.9^{\circ}$ kyphosis and almost complete resolution of the abscess.

\section{Discussion}

Treatment of MDR tuberculosis remains a challenge, as the number of cases are on the rise, particularly in developing countries. There are multiple causes for developing resistance to tuberculosis, and some papers have described MDR-TB as a 'man-made' disease [4,5].

The risk factors for developing resistance have been reported as young age (25-44 years), female gender, patients with human immunodeficiency virus infection, previous treatment for tuberculosis, or are in contact with a person with MDR-TB [14]. In our study, the patients were young, with a mean age of 29 years, and although there was no sex predilection, $77 \%$ had been previously treated for tuberculosis.

WHO recommends the use of the Xpert MTB/RIF assay to detect extrapulmonary tuberculosis [15] and as a replacement test for other non-respiratory specimens. However, the latter is a conditional recommendation based on low-quality evidence [2]. The advantages of using the Xpert MTB/RIF assay are early detection of tuberculosis ( $<2$ hours) [16] and its ability to detect RR. In this study, only 43\% (13 of 30) of patients were diagnosed with Xpert assay because of its availability. Nevertheless, the sensitivity and specificity of the Xpert MTB/RIF assay to detect RR in our study was $100 \%$ and $92.3 \%$, respectively. These findings were similar to those obtained by Held et al. [17].

Importantly, a significant reduction in the time interval between initial presentation at the hospital and diagno- 
sis of disease was noted. For patients diagnosed with the Xpert MTB/RIF assay, therapy was initiated almost 7 months (225 days) earlier than those patients in whom the assay was not done. Early detection of MDR-TB aids in prompt initiation of the appropriate ATT, which in turn significantly reduces devastating neurological complications and permanent disabilities due to MDR tubercular spondylodiscitis [18]. Early detection also helps prevent the gradual transformation of drug-susceptible tuberculosis to a drug-resistant epidemic state, particularly in "high MDR-TB burden' countries such as India.

WHO has outlined specific recommendations for the treatment of drug resistant pulmonary tuberculosis. However, until recently, there were no particular guidelines for the treatment of drug resistant 'extrapulmonary' tuberculosis. In 2014, WHO recommended that patients with drug resistant 'extrapulmonary' tuberculosis to be treated using the same protocol as that of drug resistant pulmonary tuberculosis [19]. All patients in this study were treated according to the WHO protocol for drug resistant pulmonary tuberculosis after drug susceptibility testing. In total, $87 \%$ (26 of 30 ) of patients completed a 2 -year treatment plan with the appropriate second-line ATT and were cured of the disease.

Drug toxicity was encountered in approximately one third of the patients. Managing the adverse effects of the drugs used to treat MDR-TB can be challenging. However, failure to address these problems can result in poor adherence and an opportunity for tuberculosis to develop further resistance. Adverse effects in a similar cohort of MDR-TB patients included ototoxicity (41.8\%), psychiatric disorders (21.3\%), gastrointestinal disturbance (14.0\%), arthralgia (11.4\%), epileptic seizures (9.9\%), hepatitis (4.5\%), and dermatological effects (4.5\%) [20]. In contrast, patients in this cohort primarily developed hypothyroidism due to ethionamide and ototoxicity due to aminoglycoside therapy. Central nervous system toxicity was not encountered in our study.

Close monitoring of patients is necessary to ensure that adverse effects of second-line drugs are promptly recognized. Although the patient can reliably report most adverse effects, it remains important to have physicians trained to screen patients regularly for the symptoms of common adverse effects.

The cost per patient analysis of MDR patients revealed 70 times increase when compared to that of conventional 1st line anti-tuberculous treatment. This finding is compa- rable to that in the $2015 \mathrm{WHO}$ report [21]. Cost analysis excluded the cost of surgery, surgical implants, hospital consultations, investigations, drug-related complications and patients travel expenses.

\section{Conclusions}

Prevalence of MDR tubercular spondylodiscitis was 4.9\%. The sensitivity and specificity of the Xpert assay for rifampicin resistance was $100 \%$ and $92.3 \%$, respectively. The average time to initiation of second-line ATT for MDR patients was decreased by 7 months when the Xpert MTB/ RIF assay was used as a diagnostic tool.

\section{Conflict of Interest}

No potential conflict of interest relevant to this article was reported.

\section{References}

1. World Health Organization. Use of high burden country lists for TB by WHO in the post-2015 era [Internet]. Geneva: World Health Organization; 2015 [cited 2017 Nov 24]. Available from: http://www.who. int/tb/publications/global_report/high_tb_burdencountrylists2016-2020.pdf.

2. World Health Organization. Global tuberculosis report 2018 [Internet]. Geneva: World Health Organization; 2018 [cited 2018 Nov 27]. Available from: http://www.who.int/tb/publications/global_report/ en.

3. World Health Organization. XpertMTB/RIF assay for the diagnosis of pulmonary and extrapulmonary $\mathrm{TB}$ in adults and children: policy update. Geneva: World Health Organization; 2013.

4. Jain AK, Dhammi IK, Modi P, Kumar J, Sreenivasan R, Saini NS. Tuberculosis spine: therapeutically refractory disease. Indian J Orthop 2012;46:171-8.

5. Pawar UM, Kundnani V, Agashe V, Nene A, Nene A. Multidrug-resistant tuberculosis of the spine: is it the beginning of the end?: a study of twenty-five culture proven multidrug-resistant tuberculosis spine patients. Spine (Phila Pa 1976) 2009;34:E806-10.

6. Carlsson AM. Assessment of chronic pain: I. aspects of the reliability and validity of the visual analogue scale. Pain 1983;16:87-101. 
7. Konstam PG, Blesovsky A. The ambulant treatment of spinal tuberculosis. Br J Surg 1962;50:26-38.

8. Maynard FM Jr, Bracken MB, Creasey G, et al. International standards for neurological and functional classification of spinal cord injury. American Spinal Injury Association. Spinal Cord 1997;35:266-74.

9. Sundararaj GD, Babu N, Amritanand R, et al. Treatment of haematogenous pyogenic vertebral osteomyelitis by single-stage anterior debridement, grafting of the defect and posterior instrumentation. J Bone Joint Surg Br 2007;89:1201-5.

10. World Health Organization. Guidelines for the programmatic management of drug-resistant tuberculosis: emergency update 2008. Geneva: World Health Organization; 2008.

11. World Health Organization. Guidelines for the programmatic management of drug-resistant tuberculosis: 2011 update. Geneva: World Health Organization; 2011.

12. MacNab I. Negative disc exploration: an analysis of the causes of nerve-root involvement in sixty-eight patients. J Bone Joint Surg Am 1971;53:891-903.

13. Rathinavelu B, Arockiaraj J, Krishnan V, Amritanand $\mathrm{R}$, Sundararaj GD. The extended posterior circumferential decompression technique in the management of tubercular spondylitis with and without paraplegia. Asian Spine J 2014;8:711-9.

14. Balaji V, Daley P, Anand AA, et al. Risk factors for MDR and XDR-TB in a tertiary referral hospital in India. PLoS One 2010;5:e9527.
15. Denkinger CM, Schumacher SG, Boehme CC, Dendukuri N, Pai M, Steingart KR. Xpert MTB/RIF assay for the diagnosis of extrapulmonary tuberculosis: a systematic review and meta-analysis. Eur Respir J 2014;44:435-46.

16. Boehme CC, Nicol MP, Nabeta P, et al. Feasibility, diagnostic accuracy, and effectiveness of decentralised use of the Xpert MTB/RIF test for diagnosis of tuberculosis and multidrug resistance: a multicentre implementation study. Lancet 2011;377:1495-505.

17. Held M, Laubscher M, Zar HJ, Dunn RN. GeneXpert polymerase chain reaction for spinal tuberculosis: an accurate and rapid diagnostic test. Bone Joint J 2014;96-B:1366-9.

18. Jain AK. Treatment of tuberculosis of the spine with neurologic complications. Clin Orthop Relat Res 2002;398:75-84.

19. World Health Organization. Companion handbook to the WHO guidelines for the programmatic management of drug-resistant tuberculosis. Geneva: World Health Organization; 2014

20. Torun T, Gungor G, Ozmen I, et al. Side effects associated with the treatment of multidrug-resistant tuberculosis. Int J Tuberc Lung Dis 2005;9:1373-7.

21. World Health Organization. Global tuberculosis report 2015 [Internet]. Geneva: World Health Organization; 2016 [cited 2017 Nov 24]. Available from: http://www.tbonline.info/posts/2015/10/28/globaltuberculosis-report-2015. 